\title{
Division internationale du travail, propriété intellectuelle et développement à l'heure du capitalisme cognitif
}

\section{International division of labour, intellectual property rights systems and development in the cognitive capitalism}

\author{
Carlo Vercellone \\ MATISSE-ISYS \\ Université Paris 1 Panthéon-Sorbonne \\ 106-112, boulevard de l'Hôpital \\ 75647 Paris Cedex 13
}

\section{Résumé}

Cet article se propose de repenser la problématique du développement à l'aune des transformations liées à la crise du capitalisme industriel et à la transition vers un "nouveau capitalisme », qualifié de capitalisme cognitif. Pour ce faire, l'analyse s'articule en trois parties. La première est consacrée à la crise structurelle du paradigme industrialiste légué par les théories de développement des années 1950-1980. La seconde traite des contraintes et des enjeux résultant de la mise en place d'une nouvelle division internationale du travail (DIT) fondée sur des principes cognitifs et le renforcement des droits de propriété intellectuelle. Enfin, la troisième cherche à identifier certains traits d'une stratégie de développement post-industrielle. Par un renouvellement de l'approche en sections productives, nous définissons l'idéal-type d'un modèle de développement socialement et écologiquement soutenable.

(c) 2004 Lavoisier, Paris. Tous droits réservés.

Adresse e-mail : vercello@univ-paris1.fr 


\begin{abstract}
The aim of this paper is to provide a basis for rethinking the approach of development through the changes linked to the crisis of industrial capitalism and the ongoing transition toward a "cognitive capitalism". The analysis is organised in three parts. The first one sketches the structural crisis of the industrialist paradigm inherited from development theories during the period 1950-1980. The second part stresses the constraints and challenges implied by the new shape of international division of labour based on cognitive principles and on the strengthening of intellectual property rights' systems. Finally, the third part tries to identify certain features of a post-industrial development strategy based on a socially and ecologically sustainable development model.
\end{abstract}

(C) 2004 Lavoisier, Paris. Tous droits réservés.

Mots clés : Capitalisme cognitif ; développement ; division internationale du travail ; Droits de propriété intellectuelle; sections productives.

Keywords: «Cognitive capitalism »; development; international division of labour; intellectual property rights; «productive sections ».

\title{
1. Introduction 1
}

Avec la crise du modèle fordiste, le capitalisme est entré, à l'échelle mondiale, dans une phase de métamorphose et d'instabilité majeure dont il est encore difficile d'entrevoir l'issue. Au cœur de cette grande crise de mutation, se trouve sans doute ce qu'il est convenu d'appeler la «crise du développement». Celle-ci se décline aussi bien sous la forme d'une crise de l'économie du développement (comme branche hétérodoxe de la théorie économique) que sous celle des politiques à travers lesquelles les pays du Sud ont tenté de mettre en œuvre, durant les années 1950-1980, une stratégie de rattrapage du modèle industriel des pays développés. Les tentatives de surmonter cette crise et d'offrir une alternative aux ravages des politiques d'ajustement structurel, ont permis une floraison de travaux dont l'objet fut de poser les jalons d'une nouvelle économie du développement. Il en a résulté un débat qui demeure pourtant, à notre sens, largement tributaire d'une vision essentiellement industrialiste de la dynamique du capitalisme et des concepts de développement et de sous-développement. L'hypothèse que nous nous proposons d'étayer dans cet article est que le concept de développement doit être repensé à l'aune des transformations liées à la crise du capitalisme industriel et à la transition vers un «nouveau capitalisme », qualifié de capitalisme cognitif.

Pour cette démarche, notre analyse s'articulera en trois parties. Dans la première, nous resituerons le débat actuel sur la question du développement au regard de la dynamique longue du capitalisme et d'une nouvelle configuration dans laquelle la mobilisation et l'appropriation des connaissances deviennent de plus en plus l'enjeu clé de la valorisation des capitaux et des formes d'organisation de la production. Dans la deuxième partie, nous nous pencherons sur les contraintes et les enjeux résultant de la mise en place d'une nou-

\footnotetext{
${ }^{1}$ Je remercie les referrées de la revue pour leurs suggestions et remarques critiques.
} 
velle division internationale du travail (DIT) fondée sur des principes cognitifs et le renforcement des droits de propriété intellectuelle. Dans la troisième nous montrerons comment les contradictions et les conflits qui traversent le capitalisme cognitif permettent de concevoir certains «contours » d'une stratégie de développement post-industrielle porteuse d'un nouvel universalisme qui ne soit pas celui d'une nouvelle version de l'occidentalisme, mais le résultat de la reconnaissance et de la rencontre de la pluralité de savoirs qui, dans l'économie monde, participent à la création de richesse et à l'innovation économique et sociale. Cette démarche nous permettra de définir, par un renouvellement de l'approche en sections productives, l'idéal-type d'un modèle de développement socialement et écologiquement soutenable. Il sera caractérisé par l'association d'une agriculture respectueuse de la biodiversité et l'épanouissement d'une économie fondée sur la connaissance libérée des entraves résultant de l'actuelle régulation du capitalisme cognitif.

\section{Développement et sous-développement entre théorie et histoire}

Le développement et le sous-développement sont des concepts qui évoluent et dont l'histoire est façonnée par les conflits et les mutations de la DIT. La polarisation développement/sous-développement plonge ses racines, bien avant la révolution industrielle, durant la période d'expansion du capitalisme mercantiliste, entre les $\mathrm{XVI}^{\mathrm{e}}$ et XVIII ${ }^{\mathrm{e}}$ siècles. À cette époque la formation des États-Nations européens, sur la base de l'alliance des Princes et des Marchands, s'appuie sur deux processus indissociables: l'assujettissement des mondes extra-européens et la mise en place d'une DIT structurée autour du circuit du commerce triangulaire et du système colonial des plantations.

Comme le rappelle F. Braudel (1979), l'impérialisme, à l'instar de la tendance au monopole, n'est pas un stade tardif du développement capitaliste, mais un trait consubstantiel à la formation des États-Nations et de l'économie-monde européenne.

Le clivage initial « Nord-Sud » jouera un rôle clé dans l'accumulation primitive du capital et dans l'essor de la première révolution industrielle. Dès lors, le développement du capitalisme industriel approfondira les asymétries de la DIT en leur donnant un caractère auto-entretenu et cumulatif. Il fixera, du moins jusqu'à l'après deuxième guerre mondiale, les termes de «l'ancienne» division du travail Nord-Sud, fondée sur l'échange produits manufacturiers/produits primaires.

C'est dans ce cadre que dès le XIX ${ }^{\mathrm{e}}$ siècle, sont posés, avec Ricardo, les jalons de la théorie libérale des avantages comparatifs et son corollaire, le sous-développement comme retard. L'une de ses fonctions idéologiques sera justement de faire apparaître cette division internationale du travail comme le résultat spontané d'un principe de complémentarité en masquant les rapports de force, les relations asymétriques et les effets de domination entre pays.

Durant l'après deuxième guerre mondiale, les mouvements de libération nationale et le processus de décolonisation seront le début d'une remise en cause de l'ordre impérialiste et de la DIT issue de la première révolution industrielle. Des textes fondateurs de la CEPAL jusqu'aux théories radicales de la déconnexion, c'est aussi l'âge d'or de la formation de la théorie de la dépendance et d'une économie du développement hétérodoxe. Elle fait de l'État-Nation de la périphérie, un État-Nation imitant et s'opposant à la fois au 
modèle eurocentriste, le vecteur principal du développement et de la rupture avec la DIT traditionnelle. Il s'agit d'un projet d'indépendance économique nationale capable d'assurer la transition d'un modèle dépendant vers un modèle autocentré semblable à celui des pays avancés.

Notons que la revendication du « développement», incarnée notamment par le manifeste issu de la conférence de Bandung en 1955, tout en parvenant à construire le concept et l'unité du Tiers-monde face aux deux blocs, révèle aussi ex post toute son ambiguïté. En fait, le développement coïncide avec le choix d'adopter un modèle de progrès industriel «selon lequel chacun aurait reconstruit lui-même en suivant l'exemple de l'Occident moderne colonisateur, mais sans devoir subir l'assujettissement et l'exploitation implicite au colonialisme » (Shiva, 2002a, p. 11). Cette ambiguïté conduit à importer, avec l'idée de développement, l'ensemble des catégories (concernant la richesse, les besoins, la productivité, le rapport à la nature et aux savoirs) élaborées par l'économie politique en occident dans le cadre spécifique du capitalisme industriel.

En dépit de l'opposition à la théorie libérale du retard, la plupart des courants de l'économie du développement finiront ainsi par partager un même culte de la croissance industrielle conçue comme synonyme du développement. Ce biais va de pair avec une approche dominante de la neutralité de la science et de la technologie qui en sous-estime le marquage social (Dockès et Rosier, 1988) et partant l'impossibilité d'importer le progrès technique sans reproduire les rapports sociaux incorporés dans les biens d'équipement et l'ingénierie qui organisent le procès de travail (Perrin, 1984).

Au début des années 1980, le choc monétariste et la crise de la dette mettent fin aux illusions concernant la «mondialisation du fordisme » s'étendant à la périphérie grâce au jeu combiné des délocalisations des pays du Nord et des politiques volontaristes de développement au Sud. Certes, l'espoir d'une généralisation du modèle de la production/consommation de masse s'était déjà heurté à des limites endogènes résultant, comme au Brésil, de l'inégalité de la répartition des revenus, de la dépendance technologique, des coûts croissants des politiques de substitutions aux importations dès lors que l'on passe de la section des biens de consommation à celle des biens d'investissements. Cependant, le poids croissant de la dette «étrangle les fordismes périphériques » tout en les poussant dans une course effrénée vers un modèle exportateur et hyperproductiviste qui désarticule davantage leurs économies et accélère le rythme de déstabilisation écologique de la planète (Lipietz, 1985 et 1993). Sous l'égide du FMI et de la Banque Mondiale, l'approche libérale, dans une version largement monétariste, retrouve une domination sans partage. Le paradigme développementaliste cède ainsi la place au paradigme du «Consensus de Washington » structuré autour du triptyque austérité-privatisations-libéralisation (Stiglitz, 2002). Le développement dans ce cadre n'est plus le but recherché. Il est le fruit promis d'une croissance spontanée transmise par le marché mondial et une spécialisation fondée sur les avantages comparatifs. Force est de constater que les politiques néo-libérales se sont non seulement révélées incapables de résorber le fardeau de la dette (celle-ci s'est même considérablement alourdie), mais elles ont aussi précipité une grande partie des pays soumis à l'ajustement structurel (y compris les économies en transition) dans une véritable spirale perverse de développement du sous-développement. De plus, la crise financière des années 1997-1998 a montré que la libéralisation financière peut aussi conduire à une déstabilisation des modes de développement les plus dynamiques tels ceux des pays du Sud- 
Est asiatique ayant suivi, du moins jusque vers le milieu des années 1990, des stratégies de croissance hétérodoxes (Boyer, 2001). Last but not least, l'essor des mouvements altermondialistes, la crise financière et l'embrasement social en Amérique Latine, l'enlisement de l'Afrique dans le cercle vicieux de la déconnexion forcée, viennent «couronner » la crise du consensus de Washington. Les questions indissociables du développement et de la régulation de l'économie mondiale se trouveraient ainsi, aux dires même de l'ancien économiste en chef de la Banque Mondiale, «à la croisée des chemins, exactement comme pendant la Grande Crise » (Stiglitz, 2002, p. 319).

\subsection{Renouveau et difficulté du débat actuel sur la «crise du développement »}

Il existe aujourd'hui un large accord autour du constat selon lequel nous sommes en présence d'une «crise du développement». Celle-ci est essentiellement lue à travers un prisme qui, au sens de K. Polanyi, l'appréhende comme l'expression d'un mouvement de balancier historique opposant l'État et le Marché en tant que principes de coordination économique. Dès lors, après la faillite des politiques libérales, l'enjeu du renouveau de l'économie du développement se trouverait soit dans le retour en force de l'État développementaliste, soit dans une synthèse théorique nouvelle prônant un «équilibre bien tempéré entre interventions publiques et ajustements décentralisés », comme le remarque Boyer (2001, p. 25). Cette tentative de synthèse est également au centre de la thématique de la «bonne gouvernance » développée par la Banque Mondiale à la suite d'un bilan critique des politiques d'ajustement structurel. Dans cette démarche, elle fait également appel au rôle clé de la «société civile » et au «développement participatif » en tant que niveaux intermédiaires entre l'État et le marché. Cependant, ces notions sont très floues et le rôle de la société civile est évoqué dans une logique qui, en continuité avec les politiques antérieures, vise à justifier le retrait de l'État et à « pallier les conséquences de la crise et de l'ajustement structurel » (Treillet, 2002, p. 131).

$\mathrm{Au}$ total, on raisonne comme si la question du développement se trouvait essentiellement au niveau du mode de régulation (par l'État ou le marché) susceptible de relancer une dynamique de croissance (fordiste ou post-fordiste) centrée sur le rôle moteur du capitalisme industriel ; cette vision étant plus ou moins amendée par la prise en compte des contraintes écologiques qui conditionnent la possibilité d'un développement durable ou soutenable.

Cette approche fait l'objet d'une critique radicale de la part du courant dit du « refus du développement » ou de «l'anti-développement » (Rist 2001, Latouche 1986, 2002). Ce courant, au nom de considérations opposées à celles du FMI ou de la Banque Mondiale, aboutit à la conclusion de l'impossibilité d'un développement autre que le développement réellement existant, porteur de la logique productiviste destructrice de l'environnement et d'une dépendance technologique, culturelle et financière accrue vis-à-vis de l'occident capitaliste. Le développement assimilé à la croissance est ainsi opposé au modèle culturel des sociétés traditionnelles unifiées par leur caractérisation «en négatif » face à l'économisme et à l'utilitarisme de la culture occidentale. Il en résulte une démarche qui reste linéaire. L'histoire, aussi bien au Nord qu'au Sud, n'est ni un processus linéaire (comme celui décrit par la théorie des étapes de la croissance) ni la quête d'un éternel retour (vers la culture des sociétés d'avant la colonisation), mais un processus dynamique fait de conflits et d'hybridations. L'approche de l'anti-développement a sans doute raison de pointer 
le doigt sur le productivisme implicite dans la plupart des théories du développement et d'insister sur la nécessité, du moins pour ce qui concerne les pays développés, de s'engager résolument dans la voie d'une décroissance de la production matérielle. Elle a également le mérite de revaloriser, à sa manière, le rôle que les savoirs traditionnels pourraient jouer dans l'élaboration d'une alternative au développement réellement existant, quoiqu'en tendant parfois à les enfermer dans une opposition rigide à toute forme de savoir et d'innovation technique et sociale issue des sociétés occidentales. De cette sorte, le rejet du productivisme risque, par exemple, d'englober hâtivement celui des institutions du Welfare State qui, à la suite d'une dynamique complexe conflits-innovations, ont permis durant les années 1960-70, d'introduire certains éléments de resocialisation de l'économie tout en posant les bases d'une économie fondée sur la connaissance et la primauté du non marchand.

Finalement, la réflexion sur le développement demeure tributaire d'une vision essentiellement industrialiste et n'est pas rapprochée des changements profonds qui, depuis la crise du fordisme, caractérisent l'accumulation du capital, les modèles productifs et la division internationale du travail.

\subsection{Origine, sens et enjeux de l'hypothèse du capitalisme cognitif}

Notre hypothèse est que le concept de développement doit être revu à la lumière des mutations liées à l'épuisement de la dynamique longue du capitalisme industriel et à la transition vers ce que l'on peut qualifier de capitalisme cognitif. Cette transition trouve son origine dans la diffusion et le rôle moteur du savoir engendré par la hausse du niveau moyen de formation et la montée du travail immatériel et intellectuel. Aussi la principale source de la valeur réside-t-elle toujours davantage dans les savoirs, et non dans les ressources et le travail matériels. Dans les pays capitalistes développés, la part du capital dit immatériel et intellectuel et des activités à haute intensité de savoir (services informatiques, R\&D, enseignement, formation, santé, multimédia, logiciels...) s'affirme désormais comme une variable clé de la croissance et de la compétitivité des nations (Plihon, 2001). Nous avons là une mutation, et ceci est fondamental, qui se manifeste dans l'ensemble de l'économie par l'importance des externalités liées au savoir et ses effets sur l'organisation de la division technique et sociale du travail, comme l'atteste la diffusion des tâches de production de connaissances et de traitement de l'information dans tous les secteurs économiques, y compris ceux à faible intensité technologique (Eliasson, 1996). En somme, la source de la richesse des nations se trouve de plus en plus dans l'articulation de l'ensemble des temps sociaux qui contribuent à la production et à la reproduction sociale. Dans une économie fondée sur la connaissance, le système de formation et de recherche ainsi que le temps dit « libre » comptent au moins autant que la sphère du travail salarié et de l'univers marchand. Les frontières conventionnelles entre travail et non travail s'effritent et la société dans son ensemble devient la source d'un progrès technique exogène aux entreprises. Le capitalisme contemporain se caractériserait ainsi par une remise en cause de la tendance à la polarisation des savoirs propre au capitalisme industriel et par l'affirmation d'une nouvelle prépondérance qualitative des connaissances vivantes incorporées au travail par rapport aux savoirs formalisés incorporés au capital et à l'organisation des firmes (Vercellone, 2003a, 2003b, 2003c, Herrera et Vercellone, 2003). 
Trois processus hautement conflictuels sont à l'origine de la crise sociale du rapport salarial fordiste et de la transition vers le capitalisme cognitif :

- La contestation de l'organisation scientifique du travail et du productivisme. Le refus du travail parcellisé et la montée du besoin d'autonomie des salariés vont ébranler, du moins à l'échelle des grandes usines d'OS, les formes traditionnelles de mise au travail tayloriste. Cette dynamique ira par ailleurs de pair avec le rejet d'une idée de progrès assimilée à la logique de produire toujours plus, comme en témoigne aussi, en France, la contestation des effets de la modernisation de l'agriculture impulsée par la $\mathrm{PAC}$ et l'essor de la réflexion sur le projet d'une « agriculture paysanne » (Herman, 2002);

- La constitution d'une intellectualité diffuse issue du phénomène de la " démocratisation de l'enseignement » et de l'élévation du niveau général de formation (Vercellone, 1999). Cette nouvelle qualité intellectuelle de la force de travail conduit à la remise en cause des formes de la division du travail et du progrès technique propres au capitalisme industriel ;

- L'expansion des dépenses de l'État-Providence. Cette dynamique a contribué à la crise du fordisme par l'atténuation de la contrainte monétaire au rapport salarial et la hausse des coûts de reproduction de la force de travail (Aglietta, 1976). A posteriori, nous pouvons affirmer qu'elle a posé, en même temps, les jalons d'une économie fondée sur la connaissance, selon une logique qui, sur bien des aspects, pourrait constituer une alternative à l'actuelle régulation du capitalisme cognitif.

En somme, la crise du capitalisme industriel est dans une large mesure le résultat d'une dynamique de transformation sociale qui a dessiné les contours d'un modèle de développement alternatif structuré sur deux axes principaux :

- La réappropriation et la socialisation des savoirs comme vecteur d'un dépassement de l'organisation dite scientifique du travail et de l'expérimentation de formes alternatives de coopération sociale et productive rejetant le productivisme;

- L'expansion des services collectifs du Welfare State (santé, éducation, recherche, etc.) en tant que secteurs moteurs d'un mode de développement non productiviste, fondée sur la primauté du non marchand et les productions intensives en connaissance finalisées par la production de l'homme par l'homme ${ }^{2}$ et par la reproduction d'une intellectualité diffuse.

Notons que dans ces deux axes nous trouvons l'anticipation de ce mode de développement alternatif, qui selon R. Boyer (2002), par exemple, pourrait être le nouvel horizon ouvert par l'effondrement des mythes de la nouvelle économie et la crise du régime de croissance financiarisé. Un modèle qui pourrait également constituer une référence essentielle pour repenser un concept et une stratégie de développement soutenable et solidaire à même de réconcilier Nord et Sud de l'économie mondiale (en remettant en cause l'actuelle logique de régulation véhiculée par le capitalisme cognitif).

La mise en place des conditions d'une économie fondée sur la connaissance et la centralité du travail immatériel et intellectuel précède d'un point de vue logique et historique

\footnotetext{
${ }^{2}$ Pour ce concept cf. aussi Boyer (2002)
} 
la genèse du capitalisme cognitif. Celui-ci est, dans une large mesure, le résultat d'un processus de restructuration du capital qui vise à normaliser et à étouffer le potentiel d'émancipation inscrit, depuis la crise sociale du fordisme, dans l'essor d'une intellectualité diffuse et d'une économie basée sur la diffusion et le rôle moteur du savoir.

C'est aussi pourquoi l'interprétation en termes de capitalisme cognitif se différencie radicalement des approches en termes d'une économie fondée sur la connaissance (EFC) (Foray, 2000, Guellec, 2002) : la limite méthodologique majeure des approches en termes d'EFC est d'oublier que la nouveauté de la conjoncture historique actuelle consiste en une EFC soumise et encadrée par les formes institutionnelles régissant l'accumulation du capital (Lebert et Vercellone, 2004) (voir Encadré 1).

\section{Encadré 1 Capitalisme cognitif et économie fondée sur la connaissance : les mots et les choses}

La plupart des approches en termes d'économie de la connaissance sont caractérisées par une vision a-historique, positiviste et non conflictuelle de la science et de la technologie qui conduit à effacer les contradictions sociales, éthiques et culturelles que le développement de l'économie du savoir engendre. En un certain sens, ce type d'approche, notamment dans la littérature issue de l'OCDE, évacue la dimension capitalistique qui encadre et, à notre sens, risque d'étouffer les ressorts mêmes d'une économie fondée sur la connaissance. L'approche en termes de capitalisme cognitif, quant à elle, s'oppose à cette démarche réductrice en mettant l'accent sur l'historicité des économies et sur les conflits de savoir et de pouvoir qui vont de pair avec le développement d'une économie fondée sur la connaissance.

C'est la raison pour laquelle nous insistons sur les deux termes dont se compose le concept de capitalisme cognitif (CAPITALISME + COGNITIF). Ils mettent en exergue la dimension historique et la dialectique conflictuelle entre les deux termes qui composent ce concept, c'est-à-dire :

i) le terme capitalisme désigne la permanence, dans le changement, des invariants fondamentaux du système capitaliste : en particulier le rôle moteur du profit et le rapport salarial ou plus précisément les différentes formes de travail dépendant sur lesquelles repose l'extraction du surplus.

ii) le terme cognitif, quant à lui, met en évidence la nature nouvelle du travail, des sources de la valeur et des formes de propriété sur lesquelles s'appuie l'accumulation du capital. Il met également en évidence la nature nouvelle, elle aussi, des contradictions qui caractérisent le capitalisme cognitif.

Aussi la transition vers le capitalisme cognitif exprime-t-elle le jeu d'une complexe dialectique conflits - restructuration par laquelle le capital tente d'encadrer, de s'approprier et de soumettre à sa logique les conditions collectives de la production des connaissances.

Ainsi s'explique en grande partie la manière dont le capitalisme cognitif, sous l'égide de la finance et des politiques néo-libérales, a impulsé un nouveau processus de désocialisation de l'économie qui vise deux objectifs, en contradiction avec les conditions sociales et institutionnelles, qui pourraient permettre une gestion efficace de l'économie de la connaissance. Le premier objectif est d'élargir la sphère marchande en colonisant progressivement les institutions du Welfare State et les biens communs représentés par le savoir et le vivant. Le second est d'accentuer, par le retour en force d'un ordre concurrentiel, la précarité et l'individualisation du rapport salarial, car le renforcement de la contrainte économique sur le salariat devient une condition essentielle du contrôle et de la mise au travail d'une force de travail de plus en plus autonome au niveau de la sphère de production.

Aussi, nous pouvons affirmer que l'actuelle régulation du capitalisme cognitif repose sur une logique qui peut aller jusqu'à saper les sources collectives de la production des connaissances. De même, le capitalisme cognitif ne supprime pas la logique productiviste du capitalisme industriel pas plus que celle de la croissance des biens matériels. Au contraire, 
il les réarticule et les renforce en mettant la science et les nouvelles technologies au service d'une quête de standardisation et d'appropriation privée du vivant qui, dans la droite ligne de deux siècles de capitalisme industriel, accentue les risques de destruction de la biodiversité et de déstabilisation écologique de la planète.

La transition vers le capitalisme cognitif dans les pays développés est ainsi associée à deux autres défis majeurs qui ont un impact crucial sur la restructuration de ce qu'il est convenu d'appeler le rapport Nord-Sud et l'élaboration d'une stratégie de sortie du sousdéveloppement :

- Le premier est lié aux limites écologiques de la croissance (industrielle et matérielle) qui renversent la positivité de cette dernière (la production de masse comme lutte contre la rareté) en une force de destruction. Elle rend inconcevable une extension planétaire du paradigme capitaliste (industriel, mais aussi cognitif) de développement ;

- Le deuxième a trait à la mise en place d'une nouvelle DIT fondée sur des principes cognitifs. Elle repose, pour des pans entiers de la production, sur le passage d'une logique taylorienne de décomposition du procès de travail vers une logique d'apprentissage et de compétence. La régulation de cette nouvelle DIT va de pair, notamment sous l'impulsion des États-Unis, avec une refonte des Droits de propriété intellectuelle (DPI) qui, depuis les accords ADPIC (Accords sur la propriété intellectuelle relatifs au commerce), tend à s'étendre à l'ensemble de la planète. Elle correspond à une stratégie impériale qui vise à assurer aux États-Unis et à un cercle restreint des firmes de pays de l'OCDE les dividendes de la propriété intellectuelle, en reléguant le reste du monde dans la production de biens banalisés et l'approvisionnement en matières premières (Coriat, 2002).

\section{Capitalisme cognitif, propriété intellectuelle et nouvelle division internationale du travail}

Le processus de mondialisation actuel peut, sur bien des aspects, être interprété comme le renouvellement d'un vaste processus d'accumulation primitive. Il combine étroitement les méthodes traditionnelles de l'expropriation originelle et la tentative de transformation en marchandises de la totalité du monde de la vie et de la pensée. Ce processus prend des formes néo-colonialistes qui s'appuient notamment sur l'extension et le renforcement du système de propriété intellectuelle. Il achève le processus d'accumulation primitive, commencé par l'appropriation de la terre au temps de la première colonisation et qui se continue au travers de la privatisation des semences et des savoirs traditionnels accumulés par les populations indigènes. En ce sens, « avec le droit de propriété intellectuelle et les brevets, la piraterie s'exerce directement sur les cerveaux et sur les corps des populations indigènes : c'est la vie elle-même qui est colonisée » (Shiva 2001, p. 13).

On assiste ainsi, à une nouvelle dynamique de privatisation parasitaire du « commun » qui investit, du Nord au Sud de l'économie monde, les savoirs traditionnels comme les savoirs nouveaux de l'économie de la connaissance, les anciens droits collectifs sur les espaces agricoles et forestiers et les services collectifs du Welfare State.

Le contenu essentiel de cette refonte de l'accumulation du capital repose sur la « captation » de l'économie du savoir au moyen et au profit du financier et de la générali- 
sation d'une économie de rente. Le drainage formidable des ressources opéré du Sud vers le Nord grâce au service de la dette, comme la place centrale jouée par la finance de marché participent au même titre que l'extension du système des brevets à la mise en place d'une logique d'accumulation où les frontières entre rente et profit s'estompent ${ }^{3}$.

\subsection{Vers la mise en place d'une division cognitive du travail et une nouvelle géographie du développement}

Deux facteurs étroitement liés structurent une nouvelle DIT qui s'accompagne d'une exacerbation des inégalités spatiales de développement.

Le premier résulte « de la montée inexorable du contenu en connaissances scientifiques et techniques dans les activités productives » (Mouhoud, 2003, p. 136). Dans la mesure où le capital physique devient une variable secondaire par rapport à la capacité de mobiliser en réseau les intelligences des hommes, on assiste au basculement vers une division cognitive du travail reposant sur le « fractionnement des processus de production selon la nature des blocs de savoirs qui sont mobilisés » (ibidem, p. 127).

Dans les pays capitalistes avancés, nous assistons à la mise en place d'une nouvelle division cognitive du travail qui rompt avec les principes smithiens ayant structuré le développement du capitalisme industriel (Vercellone, 2003b et 2003c). Cette nouvelle logique de la division du travail, fondée sur des principes cognitifs, est désormais hégémonique d'un point de vue qualitatif ${ }^{4}$ par rapport à une logique néo-tayloriste flexibilisée, même s'il existe une dualité entre ces deux logiques. Cette prépondérance est attestée par la mobilité du capital : les espaces en difficultés sont de type néo-tayloriste en raison de leur vulnérabilité à l'extrême volatilité du capital. À l'inverse, les activités intensives en connaissance sont beaucoup plus ancrées territorialement puisque, dans ce cas, c'est le capital qui dépend d'un bassin de travail intellectuel et immatériel, lequel préexiste à l'activité des firmes et se concentre notamment dans les métropoles. Dans la nouvelle division cognitive du travail, le facteur déterminant de la compétitivité d'un territoire dépend de plus en plus du «stock » de travail intellectuel mobilisable de manière coopérative par celui-ci. Dans ce cadre, « la logique d'exploitation d'avantages comparatifs recule au profit de la détention, par le territoire, d'éléments de monopoles ou d'avantages absolus sur des compétences spécifiques » (Mouhoud, 2003, p. 128). La mise en place d'une division cognitive du travail va ainsi de pair avec une tendance à la polarisation nouvelle de la géographie du développement entre régions et nations, particulièrement marquée pour ce qui concerne les activités intensives en connaissance ${ }^{5}$.

\footnotetext{
${ }^{3}$ En ce sens, les hypothèses de «Capitalisme actionnarial » et de « capitalisme cognitif », loin de s'opposer, désignent une mutation unique de l'accumulation du capital. Pour une discussion concernant les convergences et les divergences entre ces deux grilles de lectures voire aussi Vercellone (ed.) (2003a) et Paulré (2003).

${ }^{4}$ Par le concept d'hégémonie, il ne faut pas ici entendre une dimension purement quantitative, mais le type de division du travail et la composition de la force de travail sur laquelle repose tendanciellement la valorisation du capital. Pour illustrer cette idée, nous pouvons songer au fait que lors de l'essor de la première révolution industrielle en Angleterre, le factory system s'est affirmé comme le modèle productif dominant, même si, d'un point de vue quantitatif, ce n'est que vers la deuxième moitié du XIX ${ }^{\mathrm{e}}$ siècle que le nombre d'effectifs employés dans les fabriques dépasse celui du putting-out system.

5 Nous caractérisons là bien entendu une tendance lourde. Nous invitons le lecteur à consulter Benko et Lipietz (2000) pour une analyse plus fine sur la «nouvelle géographie socioéconomique ».
} 
Cette tendance à la polarisation est d'autant plus forte que l'automation et les économies de variété permettent aux pays d'ancienne industrialisation de reconquérir des avantages comparatifs y compris dans les secteurs intensifs en travail. C'est pourquoi, la nouvelle DIT se caractérise également par une tendance à la relocalisation d'activités productives qui, lors de la crise du fordisme, ont fait l'objet d'une décentralisation productive dans les pays à bas salaires. Mieux encore, le brevetage du vivant et la révolution bio-technologique permettent souvent aux firmes du Nord de remplacer par des «marchandises nouvelles » les produits et les matières premières traditionnellement importées du Sud ${ }^{6}$. Certes, nous ne sommes pas là non plus, face à un processus univoque : de la même manière que certaines phases de la production peuvent être relocalisées dans des régions développées, il est possible pour certaines fonctions de direction et de conception d'être délocalisées vers des pays du Sud ou de l'ancien bloc socialiste disposant d'un important réservoir de maind'œuvre intellectuelle. Une logique de délocalisation basée sur la réduction des coûts de travail peut ainsi se combiner à la nouvelle logique de la division cognitive du travail (Lebert et Vercellone, 2003)

Il n'en reste pas moins que malgré l'augmentation considérable de l'investissement direct à l'étranger (IDE), celui-ci reste concentré dans les pays développés et dans un nombre limité de NPI à forte croissance disposant d'un vaste marché et/ou d'un fort potentiel de force de travail qualifié. Le développement inégal de l'économie de la connaissance tend ainsi à engendrer un processus autoentretenu et cumulatif qui condamne un certain nombre de pays en développement à une véritable «déconnexion forcée » (Mouhoud, 1992 \& 2002). La menace de la « déconnexion forcée » est renforcée par les barrières protectionnistes que les pays du Nord continuent à ériger, comme le montre le cas exemplaire des politiques agricoles américaine et européenne. En somme, comme le dirait Bairoch, «le libre échange continue à être un mythe » sauf pour les pays du Sud auxquels il est imposé.

\subsection{La refonte des droits de propriété intellectuelle (DPI) : les nouvelles enclosures du savoir et du vivant}

Le deuxième facteur qui structure la nouvelle DIT est représenté par les « enclosures du savoir ». Les nouvelles clôtures du capitalisme cognitif se développent notamment par une refonte des DPI qui, en distendant la frontière entre découverte et invention, autorise le brevetage du vivant et du savoir. Cette politique de constitution institutionnelle de « rentes de position » est souvent justifiée par l'argument selon lequel, dans les secteurs à forte intensité en savoir, l'essentiel des coûts est fixe et se trouve dans les investissements en R\&D des entreprises. Une fois conçus, le coût marginal de «reproduction» de ces biens et services intensifs en connaissances est en revanche très réduit ou quasiment nul, comme par exemple dans le secteur des NTIC. Ces biens devraient donc être cédés gratuitement. L'élargissement et le prolongement des DPI, permettant aux firmes d'amortir leurs coûts de R\&D, seraient alors la condition essentielle de l'innovation.

\footnotetext{
${ }^{6}$ Ce processus de substitution aux importations en provenance du Sud repose en partie sur le phénomène de la bio-piraterie (Shiva, 2002b). On peut songer, à titre d'exemple, à la manière dont les États-Unis ont construit une économie du riz états-unien d'exportation à partir de l'utilisation de variétés de riz basmati sélectionnées à l'origine par des paysans indiens, et sur lesquelles ensuite des firmes américaines, comme Rice Tec ou Pepsi, ont revendiqué des droits de propriétés intellectuels au moyens de brevets et/ou des marques.
} 
Différents arguments développés ci-après permettent de critiquer le bien fondé de cette défense théorique des DPI, en tant que mécanisme stimulant l'innovation et de mettre en exergue son rôle idéologique qui vise à justifier l'appropriation par les grandes firmes du Nord des ressources génétiques et des savoirs traditionnels du Sud.

\subsubsection{Les DPI comme facteur de blocage de la circulation des savoirs et de développement de l'économie de la connaissance}

Le renforcement du système de DPI ne repose sur aucune véritable justification économique objective ni en tant que mécanisme de stimulation de la recherche ni comme récompense d'un processus effectif de production de connaissances conduit sur une base exclusivement privée. Plusieurs observations plaident en ce sens.

a) La thèse selon laquelle la recherche du profit et du gain individuel constituerait le facteur principal d'incitation à la recherche et de l'innovation a pour origine une conception réductrice de la production du savoir lue comme un phénomène isolé et sans rapport avec le tissu social et les connaissances préalablement accumulées par la société. Elle suppose un système de recherche et d'innovation entièrement privée et marchand dans lequel le savoir ne serait destiné à remplir que trois fonctions essentielles : celles d'une marchandise pouvant être vendue, d'un capital immatériel livrant un avantage compétitif, et d'un instrument de contrôle et de verrouillage du marché (cette dernière fonction étant souvent inavouée). Cette vision occulte la manière dont en réalité la plupart des coûts fixes de recherche se trouvent en amont du système même des entreprises et de leurs centres de R\&D. En fait, les conditions sociales de la recherche et de l'innovation sont de plus en plus collectives et dépendent, en dernière instance, de la qualité et de la densité de la force de travail formée par le système d'enseignement public. De plus, un grand nombre des brevets détenus par les firmes multinationales ne sont pas le produit direct de leurs efforts de R\&D. Ils ont été au préalable le produit de recherches développées par des institutions publiques ou encore, comme dans le cas de la bio-piraterie, sont le résultat d'une « prédation » des savoirs des communautés traditionnelles.

b) La privatisation du savoir peut devenir un mécanisme de freinage de l'innovation et de la dimension cumulative liée à l'économie de la connaissance, et ce pour cinq raisons principales : dans la communauté scientifique ainsi que dans les sociétés traditionnelles, la motivation principale de la recherche n'est pas le profit, mais plutôt la reconnaissance par les pairs. Ainsi, dans un système de savoirs ouverts, le chercheur, en l'absence d'une logique de privatisation du savoir, est plutôt motivé par un comportement que l'on peut qualifier par la formule «publier à tout prix » et qui conduit ipso facto au partage et à la diffusion des connaissances. À la suite du changement actuel dans le système des brevets, les centres de recherches publics tendent à se transformer en machines à produire de la propriété intellectuelle. Dès lors, la logique de la publication est replacée par celle de «breveter à tout prix ». Aussi les brevets introduisent une logique de cloisonnement et de concurrence privative dans le monde scientifique qui tend à se faire au détriment de la coopération et d'une logique de savoirs ouverts. Si cette tendance est en partie atténuée par la mise en œuvre de réseaux privatifs, plus ou moins fermés pour cause de concurrence (Carpentier, 1998), elle risque néanmoins d'entraver la dynamique collective de la production de 
connaissances et d'absorber la créativité humaine en détruisant sa source première (Shiva, 2001).

- La thèse selon laquelle le brevet permettrait la divulgation d'un savoir qui autrement demeurerait secret est en grande partie infondée (Bessen et Maskin, 2000). En particulier, elle repose sur une confusion entre le concept d'information et celui de connaissance. Or cette distinction est essentielle: le concept d'information désigne l'ensemble de données stockées, inactives, inertes ne pouvant produire par ellemême de nouvelles informations, alors que la connaissance, elle, implique un processus cognitif d'élaboration et d'apprentissage permettant la production de nouvelles connaissances au moyen de connaissances. En ce sens, ce que les brevets livrent en réalité ce n'est pas le savoir, mais l'information, et dans la mesure où les brevets empêchent l'utilisation de cette information ils entravent l'essentiel du processus cumulatif des connaissances qui résulte justement de son utilisation et d'une imitation créatrice (ibidem) ;

- Il est fallacieux de raisonner comme si l'ensemble des inventions (et désormais des « découvertes ») brevetées résultait de la seule protection des DPI, puisqu'un grand nombre d'entre elles auraient de toute façon vu le jour même sans brevets (Mansfield, 1986). De plus, il n'existe pas de corrélation prouvée entre l'existence (et l'ampleur) des DPI et la stimulation de l'innovation. Mieux encore, dans l'industrie pharmaceutique, la raison principale qui aurait conduit à demander une protection accrue serait plutôt la nécessité de préserver des rentes de monopoles face à un rythme d'innovation qui ne cesse de baisser depuis 1975 (Pignarre, 2003). En somme, dans ce cas, le rôle du brevet aurait une justification inverse par rapport à celle défendue par les tenants du système des DPI : il s'agirait avant tout de concilier un rythme d'innovation insuffisant avec le souci des laboratoires de conserver le plus longtemps possible le monopole des anciennes innovations, en retardant la production de médicaments génériques, par exemple. De même, les travaux de Bessen et Maskin (2000) du MIT et de Clement (2003) montrent que les dispositions judiciaires prises au cours des années 1980 aux États-Unis dans le but de renforcer la protection par brevets des logiciels ont conduit à la fois à une baisse du rythme d'innovation et des dépenses en $R \& D$;

- Le système des brevets risque de diriger la $R \& D$ exclusivement vers les productions les plus rentables où existe une demande solvable, au détriment, par exemple, de la recherche de traitements pour les maladies orphelines ou pour celles qui, comme le paludisme, sévissent dans les pays en voie de développement. De manière plus générale, des pans entiers du savoir risquent d'être laissés à la marge et oubliés car ils sont difficilement brevetables et/ou échappent à la logique de court-terme de la recherche du profit ;

- Un grand nombre de brevets n'ont d'autre but que celui d'empêcher des recherches et des innovations rivales dans certains créneaux d'activités. Cette stratégie dite de brevet de «saturation» (saturation patenting) ou d' «inondation » (patent flooding) », repose sur la multiplication de brevets, parfois portant sur des connaissances de base. Elle aboutit à des «situations d'excès de privatisation, au sens où (elle) se traduit par une moindre exploitation des connaissances, un ralentissent du rythme de création de nouveaux savoirs et la formation de positions dominantes ayant des effets anticoncurrentiels » (CGP, 2002, p. 155). 
Au total, le renforcement du système des DPI se présente sur bien des aspects comme un mécanisme de blocage du mouvement de circulation et de production de connaissances. Le rôle clé qu'il joue dans le capitalisme cognitif résulte de la recherche de rentes de monopole, obtenues à travers la transformation en marchandise et la privatisation de ce patrimoine commun de l'humanité qu'est le «savoir» et le «vivant». Le brevet permet de maintenir artificiellement élevés les prix d'un grand nombre de biens et services alors que leurs coûts de reproduction sont quasiment nuls (comme dans le secteur de NTIC) ou très faibles, comme dans l'industrie pharmaceutique.

Nous avons là une situation qui contredit les principes mêmes sur lesquels les pères fondateurs du libéralisme économique ont justifié la mise en œuvre et l'efficacité d'un ordre concurrentiel. Désormais, c'est la création de la propriété qui fait apparaître la rareté. Il s'agit de ce que Karl Marx, mais aussi David Ricardo, qualifieraient d'une stratégie visant à maintenir de manière forcée la primauté de la valeur d'échange contre la richesse qui, elle, dépend de l'abondance et de la valeur d'usage.

\subsubsection{DPI et rapport Nord-Sud}

Les effets pervers de la refonte du système de DPI et des accords ADPIC sur la circulation des connaissances et l'innovation ont des conséquences encore plus retentissantes sur le développement du Sud.

Nous pouvons affirmer qu'ils renouvellent et renforcent les mécanismes de l'échange inégal par un puissant mécanisme de drainage de ressources de la périphérie vers le centre. Ce dernier se développe par deux canaux principaux.

D'une part, les coûts des transferts des technologies et des savoirs vers les pays en développement sont considérablement alourdis, notamment dans les domaines de la pharmacie, des biotechnologies et des NTIC. Le fossé Nord/Sud tend ainsi à se creuser, et ce avec des conséquences dramatiques sur la situation d'urgence sanitaires de nombreux pays.

D'autre part, l'extension du brevet au domaine du vivant repose largement sur la biopiraterie et l'appropriation gratuite, de la part des firmes du Nord, des ressources génétiques et des savoirs agronomiques et médicinaux du Sud et plus particulièrement des régions tropicales qui sont parmi celles qui subissent le plus dramatiquement les effets de la « déconnexion forcée » de la nouvelle DIT ${ }^{7}$.

Cette véritable prédation de ressources est d'autant plus grave que la conception des DPI, régi par le paradigme de la science occidentale, ne prévoit aucune forme de reconnaissance et a fortiori de protection des savoirs des communautés rurales concernant la propriété, l'usage et l'amélioration des ressources naturelles.

De surcroît, le brevetage se traduit par l'interdiction d'utiliser les semences agricoles brevetées. La propriété intellectuelle peut en fait permettre à une entreprise multinationale de s'approprier un savoir traditionnel non protégé ${ }^{8}$, en imposant ensuite son monopole sur

\footnotetext{
${ }^{7}$ Selon les estimations de Shiva (2001), les pays du Tiers-Monde subiraient un manque à gagner d'environ 300 millions de dollars en royalties pour les semences développées par les agriculteurs. En ce qui concerne les plantes médicinales, ce manque à gagner en royalty est estimé à plus de 5 milliards de dollars, et ce alors qu'en 1996 il a dû payer environ 18 milliards de dollars pour acheter des technologies soumises à brevet.

${ }^{8}$ Par ailleurs, comme le rappelle Stiglitz (2002), même si « on ne sait pas vraiment si ces brevets résisteraient à l'examen des tribunaux en cas de plainte, [...] il est clair que les pays non développés n'ont pas les moyens juridiques et financiers nécessaires pour les contester » (p. 315).
} 
la commercialisation des semences, y compris aux agriculteurs qui pratiquaient cette culture depuis des siècles.

Ce processus a deux conséquences fondamentales sur les conditions de la paysannerie et le développement de l'agriculture du Sud (mais aussi de celle du Nord) :

- Premièrement, il introduit une séparation artificielle entre l'acte de production «qui reste dans les mains du paysan (et) la reproduction qui doit devenir le monopole, le privilège du capital - maintenant un cartel d'entreprises transnationales » (Berlan, 2002, p. 206) ;

- Deuxièmement, les semences brevetées risquent de s'imposer et de remplacer progressivement les autres variétés, en poursuivant le processus d'uniformisation et de standardisation de la production agricole. Il en résulte l'imposition de monocultures qui finissent par détruire cette même bio-diversité et ce réservoir de savoirs sur lequel s'est appuyé le développement des entreprises biotechnologiques du Nord. Contrairement aux arguments utilisés par les promoteurs de la révolution verte puis des OGM, le développement de l'agriculture intensive, loin de permettre de subvenir aux besoins d'une population croissante, détériore les conditions de vie de la paysannerie, tout en la rendant de plus en plus dépendante des grandes firmes agrochimiques. En somme, les «monocultures se diffusent non parce qu'elles permettent de produire plus, mais parce qu'elles permettent de mieux contrôler », (Shiva, 1993, p. 11). Cet auteur montre également, à partir d'une synthèse de travaux statistiques récents, de quelle manière la thèse selon laquelle l'agriculture intensive à grande échelle assurerait une productivité plus élevée que celle des fermes paysannes mettant en œuvre des polycultures biologiques est un «mythe ». La mesure de la productivité de l'agriculture intensive est non seulement biaisée parce que les coûts et les externalités négatives de tous les intrants (ressources, énergies, semences) ne sont pas comptabilisés, mais aussi parce qu'on ne prend en compte qu'une partie de la production, celle vendable sur le marché (Shiva, 1993 et 2002c).

\section{Capitalisme cognitif, économie fondée sur la connaissance et développement soutenable : quelques pistes de recherche}

L'analyse du capitalisme cognitif et de la nouvelle DIT peut livrer plusieurs enseignements en vue d'un renouveau de la théorie du développement : un renouveau capable de redéfinir à la fois les moyens et le contenu du développement en les inscrivant dans le projet d'une «mondialisation autre ». Dans cette perspective, nous allons esquisser quelques axes de réflexions qui pourraient contribuer à l'élaboration d'un programme de recherche.

1) Une première série d'enseignements a trait à la critique des stratégies libérales du consensus de Washington, qu'elle permet de préciser sur au moins deux points fondamentaux :

a) La nouvelle DIT infirme, encore plus que par le passé, la thèse selon laquelle les «pays en développement» devraient se spécialiser dans les activités intensives en main-d'œuvre et à faible contenu en connaissance. Le processus de divergence cumulative endogène à l'économie de la connaissance, la réversibilité des processus de délocalisation rendue possible par les NTIC, sans oublier la capacité des biotechnolo- 
gies de créer des produits remplaçant les anciennes matières premières importées auparavant du Sud, font en sorte que ce type de stratégie d'insertion dans la DIT risque de se révéler à terme comme la voie la plus rapide vers la « déconnexion forcée »;

b) Les politiques d'ajustement structurel, loin d'avoir orienté l'IDE vers les PVD, ont conduit à un recul dramatique des dépenses dans les systèmes d'éducation et de santé. De cette sorte, elles ont non seulement eu des effets néfastes sur les conditions de vie des populations, mais elles ont aussi déstructuré les pré-requis essentiels à l'essor d'une économie fondée sur la connaissance et ceux d'une possible insertion non subalterne dans la DIT.

2) Une deuxième série d'enseignements concerne l'identification des secteurs moteurs sur lesquels pourrait reposer une stratégie de sortie du sous-développement adaptée aux défis du capitalisme cognitif et de la crise du paradigme industriel du développement.

Dans cette démarche, il peut se révéler utile de partir d'une relecture critique du noyau dur commun aux théories développementalistes des années 1950-70. La problématique à la base de ces théories peut être synthétisée par une contribution essentielle de Amin (1973) qui caractérise, à travers une approche en sections productives, les modèles types respectifs d'une économie du centre, d'une part et d'une économie périphérique, d'autre part.

Le premier modèle repose sur une articulation cohérente entre la section industrielle de biens d'équipement (S1) et la section de biens consommation de masse (S2). Il s'agit donc d'un modèle autocentré, dont les relations fondamentales ont été celles décrites par l'école de la régulation par le concept de Fordisme.

À l'opposé, le régime d'accumulation d'une économie type de la périphérie repose sur un modèle extraverti et dépendant. Ce modèle est construit sur la relation fondamentale entre une section exportatrice (S3) et une section de biens de consommation de luxe (S4). Les sections industrielles modernes $\mathrm{S} 1$ et $\mathrm{S} 2$ y sont quasiment absentes. La reproduction de la force de travail, quant à elle, est assurée essentiellement par un artisanat et une agriculture traditionnels constitués par ce qui reste des sociétés périphériques d'avant la colonisation et la pénétration du capitalisme. Il s'agit en somme de «l'extérieur » (du capitalisme) de Rosa Luxemburg.

Un autre trait essentiel de l'économie périphérique est en fait le dualisme. Il se caractérise par la juxtaposition d'un secteur capitaliste extraverti et d'un secteur traditionnel archaïque et pour l'essentiel non marchand.

Le processus de développement correspond dès lors à une politique volontariste d'industrialisation permettant le passage du modèle extraverti et dualiste de la périphérie vers le modèle autocentré des pays développés. Selon la plupart des théories du développement, dans cette transition, le rôle essentiel joué par le secteur traditionnel est de favoriser la hausse du taux d'accumulation dans le secteur moderne capitaliste en garantissant la compression des salaires et une offre illimitée de force de travail. En raison même de ce rôle le secteur traditionnel est destiné à disparaître, et cette disparition progressive est considérée comme synonyme de développement ${ }^{9}$.

\footnotetext{
${ }^{9}$ Pour une critique de cette approche du développement on pourra aussi consulter avec profit les ouvrages de Shiva (1993 et 2002) et le dernier essai de Amin (2002) qui aboutissent pourtant à des conclusions assez différentes.
} 
Les contradictions et les conflits qui traversent la DIT et l'essor du nouveau capitalisme portent pourtant en leur sein les germes d'un modèle de développement soutenable et solidaire Nord-Sud. L'idéal-type de ce modèle pourrait être conçu, à travers une sorte de renversement des tendances caractérisant le capitalisme cognitif, comme la constitution d'une société de la « démocratie et de la coopération des savoirs » dans laquelle, pour le dire avec le Marx (1980) des Grundrisse, le «principal capital fixe devient l'homme lui-même » (p. 191).

Deux thèses principales étayent cette conception générale du développement.

a) La vision dualiste opposant un secteur moderne et un secteur traditionnel destiné à disparaître avec le développement devient de plus en plus caduque. Les crises écologiques locales et globales montrent que les savoirs collectifs des communautés paysannes qui ont permis l'évolution et la protection de la bio-diversité doivent être sauvegardés et revalorisés, en reconnaissant que ces savoirs ne sont pas primitifs mais appartiennent en revanche au futur (Shiva, 1993). Ce secteur traditionnel, lorsqu'il n'existe plus, est par ailleurs crée ex novo, comme c'est le cas aujourd'hui en Europe avec les méthodes d'agriculture et d'élevage biologiques et la redécouverte de la multifonctionnalité du métier de paysan. Aussi, face aux effets pervers du productivisme, la réappropriation de tâches et de savoir-faire que les paysans possédaient avant l'industrialisation de l'agriculture s'impose-t-elle comme une condition incontournable de sa conversion écologique et d'une production assurant la qualité de l'alimentation. L'expansion du secteur dit traditionnel ${ }^{10}$ devrait ainsi être ainsi considéré à la fois comme l'un des indicateurs et des objectifs essentiels d'un modèle de développement soutenable. Elle suppose la constitution d'une nouvelle agronomie issue de la synergie et de l'hybridation entre les savoirs agronomiques traditionnels et la recherche en nouvelles techniques économes en énergie et assurant le respect de la biodiversité. Cette perspective dépend en grande partie de la remise en cause du paradigme réductionniste d'une techno-science au service de la standardisation et de la manipulation marchande du vivant. Elle ne peut se faire que par l'évolution vers ce que Gorz (2004) appelle « une science plus qualitative », s'ouvrant aux exigences socio-politiques, écologiques et culturelles et aux savoirs paysans dit traditionnels porteurs d'un paradigme de la connaissance écologique et holistique des processus de la nature (Shiva, 2002a);

b) Les productions intensives en connaissances et finalisées par la production de l'homme par l'homme (éducation, formation continue, santé, $\mathrm{R} \& \mathrm{D}$, logiciels, pharmacie, etc.) doivent être considérées (davantage que les sections de biens de consommation et du capital matériels) comme la troisième et principale section productive. Le rôle jadis dévolu, dans une stratégie de sortie du sous-développement, à la pro-

\footnotetext{
${ }^{10}$ Nous gardons ici, dans une première approximation, la notion de « secteur traditionnel » pour nous opposer à l'approche canonique et industrialiste du développement qui faisait de ce secteur un segment de l'économie destiné à disparaître. Dans cette perspective, sa réhabilitation et son développement ne signifient pas, bien évidemment, le retour à une sorte de modèle bucolique antérieur au processus de mécanisation de l'agriculture. Elles renvoient en revanche à la nécessité de repenser une agriculture davantage extensive et écologique remettant en cause, y compris sur la base des connaissances scientifiques les plus modernes, la logique de l'agriculture intensive fondée sur les monocultures et la standardisation du vivant.
} 
duction de biens matériels et au capital fixe est désormais remplacé par la primauté accordée aux biens collectifs et relationnels et aux investissements immatériels permettant l'épanouissement d'une économie fondée sur la connaissance. La définition de cette nouvelle section productive repose sur le rôle moteur des services collectifs du Welfare State. Ces derniers, au lieu d'être considérés comme un coût dont le financement dépend des prélèvements effectués sur le secteur marchand, devraient être plutôt reconnus comme les secteurs clés d'un mode de développement intensif en savoir. Ils peuvent également remplacer une logique productive orientée vers les besoins solvables par une autre logique orientée vers la satisfaction des besoins essentiels et la valeur d'usage. C'est du développement de cette section productive que dépendent le rythme et la qualité du développement ainsi que la possibilité d'une insertion non dépendante dans la nouvelle DIT. Cette section détermine en fait les ressorts sur lesquels repose la « compétitivité à long terme » résultant de la capacité de mobiliser les compétences d'une force de travail à même de maitriser une dynamique de changement continu et de renouveler sans cesse des savoirs soumis à une obsolescence rapide. Ce potentiel est, à son tour, tributaire du degré de développement des institutions collectives assurant le libre accès au savoir et la formation d'une intellectualité diffuse.

Nous avons là certains éléments de ce que R. Boyer (2002) qualifie de modèle anthropogénétique « au sens général où éducation, santé et culture représentent une part déterminante de la production et plus encore façonnent le mode de vie» (p. 182). Un modèle qui permettrait de concilier les exigences d'un autre développement dans les pays du Nord et d'une politique nouvelle de sortie du sous-développement dans le Sud. En fait, comme le remarque également Boyer « pour les pays dont le développement n'est qu'embryonnaire, cette stratégie n'est pas sans intérêt puisqu'elle permet dans certains cas d'éviter les erreurs des stratégies d'industrialisation qui avaient le tort de ne considérer la production de bienêtre qu'à travers la fourniture de produits industriels » (ibidem, p. 186).

Ce modèle de développement solidaire Nord-Sud s'appuie sur une critique de la thèse traditionnelle selon laquelle le développement implique, du moins dans sa phase initiale, un approfondissement des inégalités pour favoriser l'accumulation au détriment de la consommation immédiate. Cette thèse perd une grande partie de sa justification théorique pour deux raisons principales : i) la réduction des inégalités est une condition essentielle de la diffusion du savoir et de l'essor d'une économie fondée sur la connaissance et par conséquent d'un processus de convergence entre pays riches et pays pauvres ${ }^{11}$; ii) de par leur nature, les investissements immatériels brouillent en grande partie la distinction traditionnelle entre biens de consommation et biens d'investissement ${ }^{12}$.

En résumé, sur un plan strictement économique, l'ancien objectif de développement (celui fondé sur la relation entre S1-S2), se trouverait subordonné à l'objectif prioritaire de la création d'une articulation cohérente entre deux nouvelles sections productives :

\footnotetext{
11 Sur ce point $c f$. aussi Piketty (2004).

12 Cette évolution pose de redoutables problèmes d'adéquation du système de comptabilité nationale et des politiques économique (Guellec, 2002, p. 138). Pour ne prendre qu'un exemple, les dépenses en éducation sont toujours considérées comme une consommation, alors que si l'on tirait toutes les conséquences d'une analyse en termes d'EFC, elles devraient être considérées comme un investissement social dans les hommes.
} 
- la section fondée sur les productions intensives en connaissances et les services du Welfare-State (que l'on notera S5). S5 remplit deux fonctions essentielles: celle d'assurer la compétitivité de long terme fondée sur les compétences et échappant en grande partie à la concurrence par les prix ainsi qu'à une spécialisation rigide ; celle d'autoriser la satisfaction des besoins internes et la reproduction des conditions sociales d'une société intensive en connaissances fondée, tant d'un point de vue macroéconomique que social, sur la réduction progressive des inégalités dans l'accès au savoir et dans la répartition du revenu ;

- la section correspondant au renouveau du secteur dit traditionnel (que l'on notera S6) ${ }^{13}$. Cette section comprend les productions agricoles (mais aussi artisanales) reposant sur la mobilisation de savoirs écologiques et holistiques de la nature. Le développement de cette section doit permettre de s'émanciper des effets pervers d'une agriculture extravertie et productiviste tout en réaffirmant l'objectif prioritaire de la souveraineté alimentaire. Cet objectif implique la réhabilitation des savoirs dits traditionnels et leur hybridation avec les connaissances les plus pointues provenant de la recherche scientifique afin d'aboutir à une nouvelle agronomie respectueuse de l'environnement et de la biodiversité.

L'hybridation entre ces différentes formes de savoir est l'un des axes essentiels sur lesquels peut reposer l'articulation dynamique et la synergie entre la section 5 et la section 6 (Voir Encadré 2).

C'est aussi pourquoi nous pouvons définir de manière générale le concept de développement soutenable comme un processus de substitution progressive des inputs et des finalités de la production se déroulant à deux niveaux :

- Un processus de substitution graduelle des activités intensives en connaissance et immatérielles aux activités matérielles réduisant en même temps et de manière drastique l'intensité énergétique de la production. Dans le secteur primaire ce processus se spécifie par le replacement progressif d'une agriculture productiviste au profit d'une « agriculture paysanne » basée sur la multifonctionnalité et le respect de la biodiversité, et ce grâce à l'utilisation de savoirs et de technique agronomiques permettant, comme dans les systèmes locaux traditionnels analysés par Shiva (1993), d'intégrer sylviculture et agriculture, qualité nutritionnelle de la production et régénération des ressources naturelles.

- Un processus de substitution progressive du non marchand au marchand opérée tant par le développement et la réappropriation démocratique des services collectifs du Welfare State que par l'essor d'un secteur associatif puissant. Nous avons là deux axes indissociables d'un mode de développement soutenable assurant à la fois la qualité de la vie et de l'environnement et celle de la force de travail dont dépend l'efficacité d'une économie fondée sur la diffusion et le rôle moteur de la connaissance.

\footnotetext{
${ }^{13}$ Une définition plus précise de ce secteur pose bien évidemment d'importants problèmes théoriques concernant aussi bien ses frontières que les critères de mesure de la richesse, selon une problématique qui sur bien des aspects peut être rapprochée du débat autour de concept de «multifonctionnalité agricole ». À ce propos cf. la revue critique de la littérature proposée par Nieddu (2002).
} 


\section{Encadré 2. Pour un prolongement de l'approche en termes de sections productives}

L'approche en section productive trouve sa référence première dans l'analyse des schémas de reproduction de Marx. Ces schémas définissent le squelette d'un mode de développement, sa cohérence macro-économique et sociale, et ce tant en ce qui concerne le bouclage du circuit que les objectifs du développement.

La partition la plus simple, proposée par Marx, est en deux sections : SI correspond à la production de biens de productions et SII à la production de biens de consommation. L'approche fondatrice de Marx en termes de schémas de reproduction a été ensuite affinée par nombre de travaux portant sur l'analyse des contraintes de l'accumulation du capital et la problématique du développement. Ainsi, dans ses travaux pionniers, Amin (1973) divise la section II en deux sous-sections : la section produisant les biens de consommation destinés à la reproduction des salariés (S2) et une section des biens de luxe pour les classes dominantes (S4). La prise en compte du commerce extérieur permet également d'identifier une section exportatrice (S3); c'est le cas du modèle d'Amin ou des travaux régulationnistes portants sur la croissance française en longue période (Bertrand, 1983). En somme, comme le rappellent Mazier et al. (1984, p. 113), « le principe de base de la classification sectionnelle est donc l'établissement d'une correspondance entre des fonctions macro-économiques, quelles qu'elles soient, et des sections, partitions correspondantes du système productif qui permettent de les assurer. Selon les fonctions retenues, peuvent donc être construits de très nombreux découpages sectionnels; aucune limite ne s'impose a priori $»$. Ainsi, comme le fait remarquer Lipietz (1985), l'identification d'autres formes ou logiques de production se reproduisant dans un rapport d'articulation avec le capitalisme et la logique marchande permet d'affiner davantage l'analyse positive ou normative des schémas de reproduction soutenant un mode de développement : on peut ainsi caractériser des sous-sections selon des modes de production et des logiques qui concourent différemment aux fonctions macro-économiques, sociales et environnementales.

C'est dans le prolongement de ces travaux que nous avons proposé d'isoler, au titre d'un programme de recherche, deux nouvelles sections productives (S5 et S6) afin d'identifier certains contours d'un mode de développement postindustriel.

En résumé, nous avons identifié trois modèles types fondés sur l'articulation déterminante entre deux principales sections productives que nous synthétisons dans le tableau ci-dessous.

\begin{tabular}{|l|c|}
\hline & Articulation centrale \\
\hline Modèle dépendant d'une économie périphérique (Amin, 1973) & S3 - S4 \\
\hline Modèle industriel d'une économie développée du centre (Amin, 1973) & S1 - S2 \\
\hline $\begin{array}{l}\text { Modèle post-industriel de développement soutenable fondé } \\
\text { sur les productions de l'homme par l'homme }\end{array}$ & S5 - S6 \\
\hline
\end{tabular}

Enfin, il convient de rappeler que la mise en œuvre de ce modèle de développement soutenable et solidaire Nord-Sud implique ce que Harribey (2002) qualifie d'un développement différencié dans son objet, dans l'espace, dans le temps, mais aussi, selon nous, dans les dispositifs institutionnels régissant la DIT :

1) dans son objet, la décroissance des productions matérielles et polluantes devrait s'appuyer sur une définition claire des domaines d'activité pour lesquelles aucune mise en valeur, et partant aucune croissance n'est souhaitable, et des domaines qui, en revanche, peuvent encore faire l'objet d'une croissance limitée ou forte ;

2) dans l'espace et dans le temps, si la décélération de la croissance matérielle est, à certaines conditions socio-institutionnelles, envisageable dans l'immédiat pour les pays développés, il en va autrement pour nombre de pays du Sud : la croissance matérielle de nombreuses productions se révèle encore nécessaire pour satisfaire les besoins d'une population en expansion démographique et pour consentir la réalisation des 
infrastructures et des biens d'équipement indispensables à la mise en place des services collectifs d'une économie fondée sur la connaissance ;

3) dans les dispositifs institutionnels de régulation de la DIT, toute stratégie de développement est quasiment inenvisageable si au moins deux conditions préalables minimales ne sont pas réunies : le retour à une configuration reconnaissant le droit pour les pays développés et les PED de mettre en place des systèmes de DPI différents (Orsi, Coriat, Weinstein, 2003); le rétablissement du droit à un protectionnisme sélectif. Celui-ci, faute de mieux, est aussi, en ce qui concerne l'agriculture, le seul instrument susceptible d'assurer, au Nord comme au Sud, la rupture avec le modèle productiviste et la mise en place de politiques nationales et régionales fondées sur l'autosuffisance alimentaire et une agriculture respectueuse de la bio-diversité. Aussi la remise en cause des accords de Marrakech, signés dans le cadre de l'OMC, concernant la libre circulation des produits agricoles et la propriété intellectuelle est-elle la voie de passage obligée de l'édification des institutions d'une mondialisation autre.

\section{Conclusions}

Pour conclure, la mutation actuelle du capitalisme et de la DIT, qualifiée de transition vers le capitalisme cognitif, est un phénomène éminemment conflictuel et contradictoire : elle accentue les effets de domination et les tendances à la polarisation de l'économie mondiale, tout en contenant en son sein la possibilité d'un modèle de développement socialement et écologiquement soutenable, basé sur la rencontre et l'hybridation de la pluralité des savoirs qui traversent l'économie monde. Nous avons tenté de caractériser certains traits de l'idéal-type de ce modèle de développement solidaire Nord-sud, tant dans son contenu économique et social que dans son contenu écologique. Il permettrait la libération du potentiel d'émancipation d'une économie fondée sur la connaissance affranchie des entraves résultant de l'actuelle régulation du capitalisme cognitif. Sa réalisation dépasse pourtant la capacité d'innovation et d'autonomie de chaque territoire et se heurte à la continuité des politiques néo-libérale de la DIT fondées sur la dérégulation des marchés et les « enclosures » du savoir et du vivant.

La crise qui secoue actuellement l'économie mondiale se présente sur bien des aspects comme la première « grande crise » de régulation du capitalisme cognitif. Une crise qui, comme le reconnaît la Banque Mondiale, montre l'impossibilité de l'actuel régime de croissance d'assurer la cohésion sociale et la préservation des ressources naturelles. Mieux encore, tout se passe comme si la crise elle-même s'affirmait comme une nouvelle forme de régulation, et certains se posent légitimement la question, à l'instar de André Gorz (2003), de savoir si le « capitalisme cognitif n'est pas un capitalisme en crise (mais) la crise du capitalisme qui ébranle la société dans ses profondeurs » (p. 82). L'issue de cette grande crise, comme l'enseigne l'histoire des autres grandes crises traversées par le capitalisme, est bien entendu indéterminée, lié au jeu d'une complexe dynamique conflits/innovation.

Quoi qu'il en soit, il est certain qu'à défaut de la constitution de formes de contre-pouvoir à l'échelle mondiale ou supranationale capable d'imposer la primauté des droits collectifs face à la logique marchande et des nouveaux droits de propriété sur le savoir et le 
vivant, la dynamique actuelle de mutation du capitalisme risque de se poursuivre selon un processus de « destruction non créatrice».

\section{Références}

Aglietta, M., 1976. Régulation et crises du capitalisme, Calmann Lévy, Paris.

Amin, S., 1973. Le développement Inégal, Édition de Minuit, Paris.

Amin, S., 2002. Au-delà du capitalisme sénile, PUF-Actuel Marx, Paris.

Benko, G., Lipietz, A., 2000. La richesse des régions - La nouvelle géographie socioéconomique, PUF, Paris.

Berlan, J.P. 2002. Sciences sous influence : le mais hybride et les mythes du progrès technique, in P. Dockès, ed. Ordre et désordres dans l'économie monde, éd. PUF, Paris, 200-244.

Bertrand, H., 1983. «Accumulation, régulation, crise : un modèle sectionnel théorique et appliqué », in Revue économique, Vol. 34, $\mathrm{n}^{\circ}$ 2, 305-343.

Bessen, J. \& Maskin, E., 2000. Sequential Innovation, Patents and Imitation, Working paper department of economics, Massachusetts Institute of Technology, $\mathrm{N}^{\circ}$ 00-01, January, 1-33.

Boyer, R., 2001. «L'après consensus de Washington: institutionnaliste et systémique », in L'Année de la régulation, $\mathrm{N}^{\circ}$ 5, Presses de Sciences Po, Paris, 13-57.

Boyer, R., 2002. La croissance, début du siècle, éd. Albin Michel, Paris

Braudel F., 1979. Civilisation matérielle, économie et capitalisme, XV-XVIII siècle, 3 Tomes, Armand Colin, Paris.

Carpentier, C., 1998. Économie du brevet. Nouveaux apports théoriques et empiriques, Thèse de Doctorat en Sciences Économiques, École des Hautes Études en Sciences Sociales, Paris.

Clement, D., 2003. « Du mythe de la nécessité des brevets pour susciter l'innovation », L'Économie Politique, $\mathrm{n}^{\circ} 19,9-24$.

Coriat B., 2002. «Le nouveau régime américain de la propriété intellectuelle», Revue d'Économie Industrielle, $\mathrm{n}^{\circ}$ 99, 17-32.

Dockès, P., Rosier, B., 1988. l'Histoire ambiguë, éd. PUF, Paris.

Dieuaide, P., Paulré, B., Vercellone C. 2003. «Le capitalisme cognitif», papier présenté aux journées d'étude MATISSE, Université de Paris 1, Avril.

Elliason, G., 1996. «Firms objectives, controls and organization», Economics of Science, Technology, and Innovation, vol. 8.

Foray, D., 2000. L'économie de la connaissance, éd. La Découverte, Paris.

Guellec D., 2002. «L'émergence d'une économie fondée sur le savoir», in J.-P. Touffut dir., Institutions et innovation: de la recherche aux systèmes sociaux d'innovation, Albin Michel, Paris.

Gorz A., 2003. L'immatériel : connaissance, valeur et capital, Galilée, Débats, Paris.

Gorz A., 2004. «Économie de la connaissance et exploitation des savoirs », entretien avec Moulier-Botang, Y. et Vercellone, C., in Multitudes, $\mathrm{N}^{\circ} 15$, janvier, Exils, Paris.

Hardt, M. \& Negri, A., 2000. Empire, Exils, Paris.

Harribey, J.M., 2002. «Développement durable : plus dure sera la chute», http://www.attac.org/fra/list/doc/ harribey9.htm

Herman, P., 2002. Changeons de politique agricole, éd. Mille Et Une Nuits, Paris.

Herrera, R., Vercellone, C., 2002. «Transformations de la division du travail et endogénéisation du progrès technique » in Économie Appliquée, Tome LV-N $N^{\circ}$-Mars 2002, Paris, 63-78.

Herrera, R., Vercellone, C., 2003. «Transformations de la division du travail et general intellect» in C. Vercellone, ed., Sommes-nous sortis du capitalisme industriel ?, La Dispute, Paris, 23-54.

Latouche, S., 1986. Faut-il refuser le développement?, éd. PUF, Paris.

Latouche, S., 2002. «L'antinomie du développement durable », in Transversales, Science, Culture, nouvelle série, 2 trimestre 2002, 10-11.

Lebert, D., Vercellone, C., 2003. «Les restructurations aux regards du capitalisme cognitif», in Régards-Les cahiers de Syndex, $\mathrm{n}^{\circ}$ 2, Novembre 2003, Paris, 32-42.

Lebert, D., Vercellone, C., 2004. «L'économie de la connaissance entre économie et histoire »,Cahiers lillois d'économie et de sociologie, L'Harmattan, Paris. 
Lipietz, A., 1985. Mirages et miracles, éd. La Découverte, Paris.

Lipietz, A., 1993. Vert Espérance, La Découverte, Paris.

Mansfield, E., 1986. « Patents and Innovation: an Empirical Study », Management Science, 32.

Marx, K., 1980. Manuscrits de 1857-1858 - Grundrisse, Tome 2, Éditions Sociales, Paris.

Mazier, J., Baslé, M., Vidal, JF., 1984. Quand les crises durent, Economica, Paris.

Mouhoud, M., 1992. Changement technique et division internationale du travail, Economica, Paris.

Mouhoud, M., 2003. « Division internationale et économie de la connaissance », in C. Vercellone, ed., Sommesnous sortis du capitalisme industriel ?, La Dispute, Paris, 121-136.

Nieddu, M., 2002. «La multifonctionnalité agricole entre marché et externalités » in Économie Appliquée, Tome LV-N¹-Mars 2002, Paris, 105-132.

Orsi, F., Coriat, B., Weinstein, O., 2003. « Brevets pharmaceutiques, efficience et équité », in Cosmopolitiques, $\mathrm{N}^{\circ}$ 5, novembre 2003, Paris.

Paulré,, B. 2003. «Postace» à l'ouvrage Sommes-nous sortis du capitalisme industriel, in C. Vercellone, ed., Sommes-nous sortis du capitalisme industriel ?, La Dispute, Paris, 329-343.

Perrin, J., 1984. Les Transferts de technologie, La Découverte, Paris.

Plihon, D., 2001. Le nouveau capitalisme, Dominos-Flammarion, Paris.

Pignarre, Ph., 2003. Le grand secret de l'industrie pharmaceutique, La Découverte, Paris.

Piketty, Th., 2004. L'économie des inégalités, La Découverte.

Rist, G., 2001. Le développement : histoire d'un croyance occidentale, Presse de la FNSP, Paris.

Shiva, V., 1993. Monocultures of de Mind. Perspectives on Biodiversity and bio-tecnology, Zeed Books Ltd., London - (1995 pour la traduction italienne, Bollati Boringhieri, Turin).

Shiva, V., 2001. Il mondo sotto brevetto, Feltrinelli, Milan.

Shiva, V., 2002a. Terra madre. Sopravvivere allo sviluppo, Utet, Turin.

Shiva, V., 2002b. La biopiraterie, ou le pillage de la nature et de la connaissance, Alias, Paris.

Shiva, V., 2002c. « La productivité des petites fermes », in l'Écologiste, $\mathrm{n}^{\circ}$.7-(vol. 3, $\mathrm{n}^{\circ}$ 1- Juin), 55-57.

Stiglitz, J.E., 2002. La grande désillusion, Fayard, Paris.

Treillet, S., 2002. L'économie du développement, éd. Nathan, Paris.

Vercellone, C., 1999. Accumulation primitive du capital, industrialisation et rapport salarial : une application au cas italien 1961-1980, Thèse, Université de Paris 8.

Vercellone, C., 2002. «Les politique de développement à l'heure du capitalisme cognitif », in Multitudes, $n^{\circ} 10$, Octobre, Exils, Paris, 11-21.

Vercellone, C., ed. 2003a. Sommes-nous sortis du capitalisme industriel, La Dispute, Paris.

Vercellone, C., 2003b. « Introduction » à l'ouvrage Sommes-nous sortis du capitalisme industriel, in Vercellone, C., ed., Sommes-nous sortis du capitalisme industriel ?, La Dispute, Paris, 7-20.

Vercellone, C., 2003c. «Mutations du concept de travail productif et nouvelles normes de répartition » in C. Vercellone, éd., Sommes-nous sortis du capitalisme industriel ?, La Dispute, Paris, 249-272.

Vercellone, C., 2003e. « Les réformes du Gouvernement Raffarin ou le capitalisme cognitif contre l'économie de la connaissance », in Archipel des Revues 3-6, disponible aussi sur le site Multitudes. 